\title{
Practicing and evaluating outcomes of working across the science policy interface
}

\author{
K.B. Matthews, D.G. Miller and D. Wardell-Johnson \\ ${ }^{a}$ James Hutton Institute, Craigiebuckler, Aberdeen, Scotland, AB158QH \\ Email: keith.matthews@hutton.ac.uk
}

\begin{abstract}
This paper reports on the practice and evaluation of co-constructed research conducted by researchers at the James Hutton Institute with a range of policy and other stakeholders. The research was undertaken to support a policy options appraisal for the reform of the Common Agricultural Policy (CAP) in Scotland, a major rural policy instrument that distributes 532M€ per annum (equivalent to over half of the total income from farming) and, by 2019 , will redistribute approximately $280 \mathrm{M} €$ of funding. Over the course of ten years of science-policy interactions a variety of modes of working have been undertaken - the paper outlines the actors, the networks of interaction, the nature of the research undertaken and how this has evolved as the research team built credibility with policy makers and other stakeholders. The increasing degree of co-construction and even co-delivery of research through partnership between James Hutton Institute staff and analysts within government is assessed in terms of the mutual benefits, the potential pitfalls and institutional and career challenges for researchers working in this way. The research was also subject to formal evaluation of policy and other impacts by consultants employed by the research funder. The paper highlights the key findings from this evaluation for the CAP related research. A key finding from the evaluation was that instrumental change (in practice or policy) was more readily acknowledged by policymakers rather than research providing new knowledge or conceptual insights. This did not match the expectations of evaluators for whom the achievement of instrumental change was the greater challenge, and was dependant on conceptual change. The paper draws on the literatures of agricultural decision support and other literatures to ground these findings in a wider community of practice.
\end{abstract}

Keywords: Co-construction, science-policy, evaluation, CAP, Scotland 


\section{INTRODUCTION}

Co-construction has become an increasingly frequently used part of the lexicon in the discourse on, and practice of, science-policy. The terminology is more immediately accessible than the alternative of transdisciplinarity but perhaps lacks the sharpness of definition that the latter implies - the going beyond academic disciplines and institutional boundaries and the sharing of power in the conduct of research. Whatever the terminology used there is the need for a clear and experientially grounded understanding of the context and practice of co-construction in the science-policy domain and evaluations of its outcomes. Otherwise, there is a danger that the term will come to be seen as yet another science-policy panacea as have, arguably and in different ways, interdisciplinarity, participation and modelling frameworks.

This paper seeks to make explicit the research team's tacit knowledge gained through experiential learning over a ten-year period of conducting research across the science-policy interface on a variety of closely related topics. The paper sets up the policy domain within which the research was conducted and the nature of the research used to support policymaking. The paper then focuses on the practice of co-construction in two parts: the phases and modes of research and the actors, networks and forums of interaction. Evaluations of the outcomes of the science-policy research are reflected on in terms of factors underpinning the success or otherwise of co-construction. The paper concludes by starting to place the empirical outcomes of this paper in a wider theoretical context, referencing both the research team's background in agricultural decision support and ideas from other literatures.

\section{BACKGROUND}

\subsection{Policy context}

The Common Agricultural Policy (CAP) is the oldest common policy of the European Union dating conceptually from the treaty of Rome in 1957, with first implementation in 1962. Its stated objectives, their balance and mechanisms have continually evolved but continue to encompass income support for farmers (70\% of budget), rural development (20\%) and market support (10\%). In 2003, payments to farmers were "decoupled" from the specifics of production with the intention that subsidy would no longer distort management decisions and that EU farming would become more market oriented and thereby World Trade Organisation compliant. The preference of the EU Commission since 2003 has been that systems of direct support payments use a single rate of payment for all land within a member state or differentiated rates for a small number of regions defined by an objective criterion such as land quality. Member States (MS) had the option of continuing to pay at historic rates in the last budget period but from 2014 the area based system became mandatory. MS do however have very considerable discretion in how these systems are implemented. Introduction of such systems of payments reduces the differences in payments between businesses with the same quality of land but differing historic intensities of production. In Scotland the CAP is a major rural policy instrument that distributes $532 \mathrm{M} €$ per annum (equivalent to over half of the total income from farming) and by 2019 will redistribute approximately $280 \mathrm{M} €$ of funding. The process of implementing the reforms was a high profile policy with the need for strong evidence to support policymaking as well as a mechanism to improve stakeholder engagement.

\subsection{Nature of the research conducted}

To support the process, an analytical framework within which the immediate consequences of area-based payment options could be assessed was built. The scope and design of this framework was generated through an iterative co-construction process between researchers in the James Hutton Institute, an independent research organisation part funded by Scottish Government (SG), analysts in the SG Rural and Environmental Science and Analytical Services Division (RESAS), who undertake in-house policy support and officials in the Rural Payments and Inspections Directorate (RPID) who administer the implementation of the CAP in Scotland. The Hutton researchers numbered three to five, with two or three RESAS analysts and one or two RPID officials. This group would be supplemented as needed for particular tasks $(<5)$. The framework combined research data on land quality; administrative data on land use, ownership and who uses the land; and data on existing payments, to serve as a baseline against which to assess the levels of redistribution. Other administrative datasets were used to define types of business (by dominant enterprise) to allow summary by sector and geographical regions within Scotland. The research tested a wide variety of regionalisation options, budgeting options (how the funds are divided between regions), eligibility (how much land is included) and activity (what has to be occurring to generate payment). These analyses were summarised in tabular, graphical and map form and used in deliberative workshop processes with policy makers and other stakeholders as discussed further in Section 3.2 and in Matthews et al., 2013. 


\section{PRACTICE OF CO-CONSTRUCTION}

\subsection{Research evolution and credibility building}

The reform of the CAP is a long-term process with some strategic changes signalled well in advance by the Commission (but with details of implementation on occasion being definitively agreed very late in the process). The transition to area-based systems of payments was the Commission's preference prior to the 2007-13 budgeting period, with a variety of degrees of transitions undertaken by MS in that period. Statements from 2011 confirmed that area based payments would be mandatory. For MS who did not transition during the 2007-13 period there was therefore a significant time window within which the options for transition could be considered. Figure 1 summarises the research team's activities during the period.

\section{Phases of research funding}

Underpinning the research are a series of five-year Strategic Research Programmes (SRP) funded by Scottish Government; these are directed research programmes with scope and resources set by government but with significant opportunity to shape research such that it delivers policy relevant outputs. The earliest SRP (200611) built Hutton researchers capacity in terms of the use of administrative datasets within research but also saw the first policy contacts via seminars presenting prototype versions of an area-based payment scheme (in 2008). Activities were also conducted through the SG Contract Research Fund - an open, competitive, project-based work stream. This saw further development of researcher expertise in the integrated use of administrative datasets (2008) and by providing support for the Pack Inquiry (2009-10). This Inquiry was a high profile assessment of options for agricultural future support, including area-based payments. A new SRP (2011-16) included further strategic CAP analysis. This was substantially supplemented by a new calldown funding mechanism in which short-term, policy-led, projects were agreed upon between SG policy leads and the researchers, mediated by RESAS (see topics in the lower half of Figure 1). Within the ten year time line (2006-2015), it is possible in retrospect to distinguish four distinct phases of co-construction between the research and policy sides (as set out below).

\section{Phases of co-construction}

From 2006 to the end of the Pack Inquiry in 2010, the modes of working moved from conventional strategic and applied research, through contract research for SG analysts and then as part of an independent inquiry team (though supervised by SG). This period saw the team build technical capability and expertise in the policy options (the likely shape of the regulations and the balance of objectives of the government). The period also generated networks of contacts from which knowledge and data could be drawn and allowed the team to refine the ways of communicating outputs. The period also established the team as a source of knowledge, particularly through participation on the Future CAP Stakeholders forum (the 60 member consultation group with members from SG policy, RIPD, RESAS, government agencies, NGOs from environment and farming, producer, service and processor representatives.. During this period coconstruction increased as the salience of the research team's analysis became apparent and the credibility of the outputs was established by their use in deliberation on policy options and public endorsement of their utility by senior officials. The research team thus continued to be included in the subsequent government led processes of policy development.

From 2011 to the Moving Forward Conference (in 2013), the degree of co-construction increased dramatically with much more regular and deeper interactions between researchers and SG staff. The new SRP in 2011 was co-constructed to a much greater degree than 2006 with significant revision to the original specifications negotiated. The revised SRP was to deliver more immediate policy support but at the same time allowed researchers a significant say in how the research questions in the SRP were organised and resourced. This period saw a series of analyses co-constructed between researchers and analysts in RESAS to address: the basis of regionalised payments; the eligible areas and how activity criteria such as stocking rates would change payments. The Moving Forward Conference marked a milestone in the process since this started a process of narrowing options from $30+$ combinations of regions and budgets.

From the Moving Forward Conference to the present, the research team continue to be actively involved in policy support undertaking a wide range of analyses both of particular policy options e.g. French Redistribution and Production coupled support and ad hoc or specialised queries such as the effects on of reforms on designated areas. In this period, the same modes of working prevailed, but as the final decision on the reform package drew closer; there was a gradual decline in the degree of involvement of the research team within SG processes. The final shape of the package of reforms was informed by analysis but political considerations immediately prior to decision-making were decisive. 
Beyond the 2014 policy decisions there have continued to be interactions with SG during the development of more detailed implementation analyses. Research within another policy-led work stream (on climate change) highlighted limitations in the administrative datasets that could have affected the new payments regime. Working with the paying agency (RPID), the team prototyped and tested new protocols for identifying potentially misclassified land. The team also provided capacity; testing options for the implementation of the regionalisation option selected (e.g. deciding how mixed land parcels should be classified). In the former case there was a great deal of co-construction, while in the latter the technical skills and datasets available to the team were used in responsive-mode testing options. This phase also saw the team tasked with supporting the development of the Greening component of CAP, the options for which were due for notification to the Commission in 2015. This was both a wide-ranging review of agriculture-environment interactions, an expert-based appraisal of the likely consequences of Greening options, and a qualifications and spatial analysis of the requirements and the degree to which they were already being met. The Greening Review scope was co-constructed between the research team and policy leads and managed by RESAS analysts. Beyond the Review the team have also addressed technical issues of Greening implementation particularly the measures applied to grasslands above those mandated by the EU (pH soil testing for all grasslands).

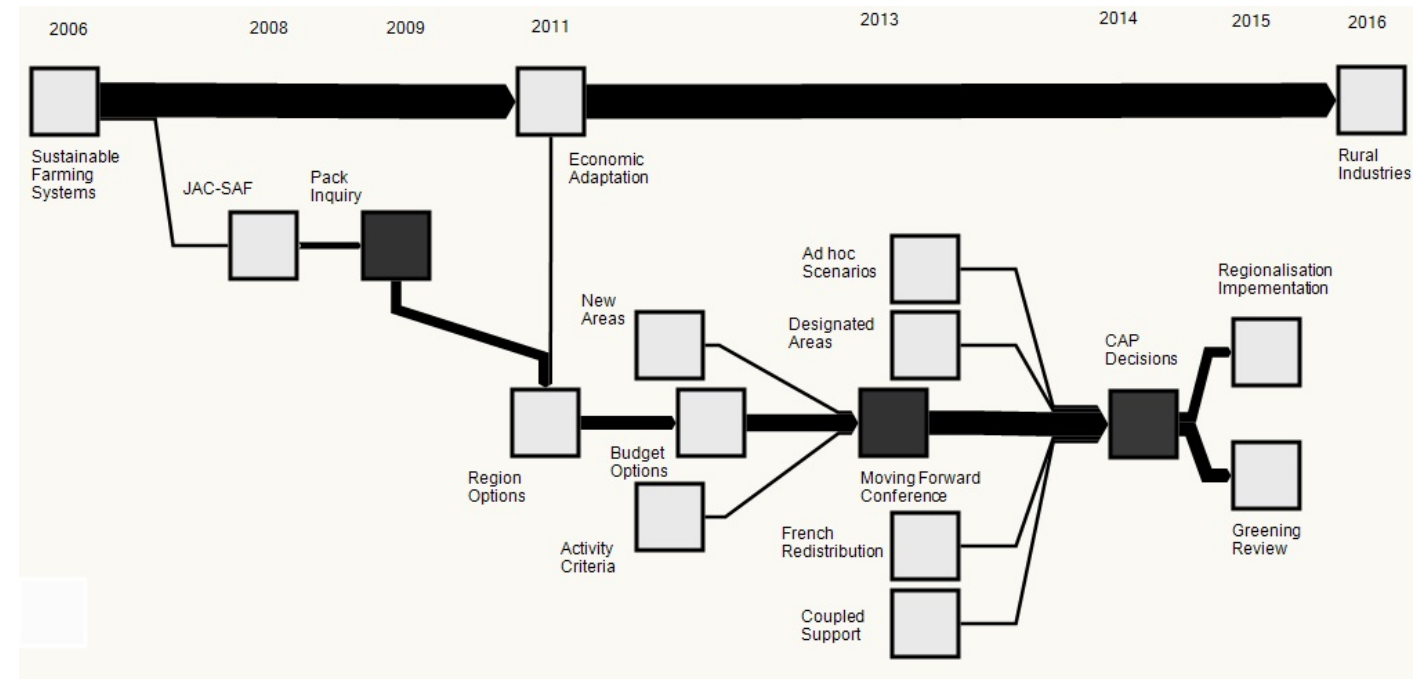

Figure 1. Work streams for CAP analysis

\subsection{Actors, networks, forums and modes of interaction}

What a work stream oriented presentation does not make clear is the number of actors with influence in the process of co-construction of the research (far less of course the policy itself). Figure 2 sets out some of the key actors and formal forums (in black) with which the research team have interacted and which shape the process of co-construction. There are of course also a great variety of informal interactions (e.g. between researchers and NGOs). The figure qualitatively represents the degree of influence on the conduct of the research in terms of co-construction by the thickness of the lines. Even in this highly simplified rendition (in most cases multiple actors are represented by a single class), the numbers of interactions are considerable with both direct and indirect influences. An example of indirect co-construction would be via the interaction between key sector NGOs and SG ministers that in turn may shape research via policy leads and RESAS analysts. This may be more influential than via channels such as the CAP stakeholders group. In the background of Figure 2 a typology of actors is presented that has been useful in planning and conducting the processes of co-construction. The implications for co-construction of each of the types is set out below.

Research Collaborators (RESAS, RPID) saw close project-based interactions on a regular basis, with faceto-face meetings, workshops and regular exchanges of data, and joint authorship or reviewing of research outputs. Co-construction of research with non-academic collaborators is a regular feature, with expertise and deliberation the basis on which any contested issues are resolved. Cordial working relationships are maintained with RESAS analysts and RPID staff (providing data and implementation expertise). Note that RESAS and RIPD staff can fulfil dual or tripartite roles as noted below.

Direct Stakeholders (SG Policy Teams, ministers) are the key groups with which there were frequent interactions either directly or mediated by third parties (RESAS analysts). These stakeholders had a directing role since they either funded or were strongly influential in the funding of research, and thus had a client and evaluative role. Direct stakeholders had their strongest influence on the initiation of research defining scope 
and objectives. They had a sign off role but were often not active in co-construction of the detail of analysis. Once the researchers had sufficient credibility, they were more influential in strategic decisions on initiation of research and definition of scope. In all cases, such groups provided the definitive policy/regulatory context within which the research was conducted (particularly the preferences of ministers). Interactions were typically though project meetings and steering groups, but also occured via multi-stakeholder advisory forums (see below). Note how RESAS acts as both a research collaborator and a direct stakeholder.

Advisers (Census Branch, Forestry Commission, Environmental Protection Agency, Natural Heritage) were the wider community of governmental branches, agencies other organisations who participated in the coconstruction of research. Interactions were less frequent and entailed data, advice on specific issues or reviews of draft research outputs. Most advisers had much less direct involvement in co-construction, but substantial indirect influence by shaping agendas either via direct stakeholders or in multi-stakeholder forums. Early interaction with this community was advantageous in allowing researchers to understand the context and narrative framing within which a specific analysis was being conducted. This is vital if there is any contestation, especially between direct stakeholders. Depending on phases of activity advisers may move been this role and that of research collaborators (e.g. RPID).

Interest Groups (National Farmers Union Scotland, Crofters Federation, RSPB) were significant sources of contextual information and expert comment on the research outputs. For this research, there were ongoing dialogues with the key NGOs listed but any co-construction had to be verified and visibly initiated via the direct stakeholders. The formal forum for interactions with advisers and interest groups was the Future CAP Stakeholders Group (meeting bi-monthly). Via this forum they were influential in shaping research agendas.

Public(s) are typically seen as less active in co-construction. Usefully included within this type, however are the members of the Scottish parliament, especially those with roles in the oversight and scrutiny of the relevant parts of the executive. They are included here since they are a key aspect of governance, as link with wider publics otherwise unrepresented and with party politics. Through interactions with ministers, MSPs influenced the research conducted e.g. initiating the CAP Greening review. Research outputs were cited in parliamentary debates and briefing materials were provided for the relevant parliamentary committee.

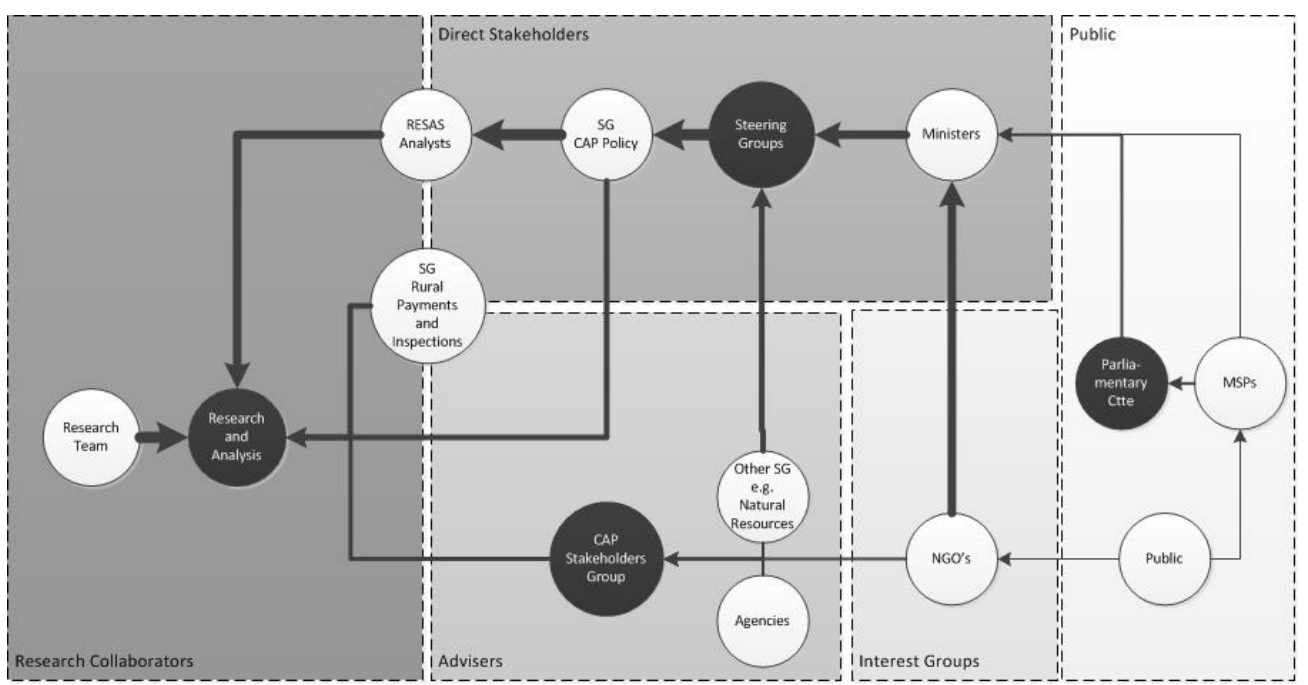

Figure 2. Co-construction network.

\section{EVALUATION}

Formal evaluation of the impacts of co-construction in science policy research presents a range of practical challenges, not the least of which are the lack of a counterfactual and the challenge of attribution. What can be reported is the researcher's perceptions of the value of co-construction based research, based on a near 10 year period conducting and refining such approaches. This can be supplemented by key findings from an Impact Review conducted by RESAS since the CAP analysis was chosen as a case study.

\subsection{Value of co-construction to the researchers}

A key strength of co-construction is generating, for the researcher a better understanding of the context of the analysis. Partly this leads to the expected increase in salience and thus utility to the client but co-construction goes beyond improving project specifications. With appropriate investment of time, it also provides the 
researcher with an appropriate lexicon with which to present results, the facility to deliver a richer interpretation of the outputs and the ability to highlight key results with implications for decision-making. Co-construction thus has a role in the gaining and maintaining of credibility.

A key weakness of co-construction is the need for a degree of continuity on key linkages (e.g. with RESAS). Short-term research project funding and turnover of civil service appointments can undermine this. Stability in terms of civil service posts, if not the individual, is essential. The political prominence and financial value of CAP meant that throughout the period there were always three RESAS staff available to support coconstruction and who were willing to draw on the range of skills and resources provided by the SRP. At each change in personnel (six in total), some of the goodwill from the previous incumbent spilled over to their replacement. This was seen in terms of readiness to engage with the research team and acknowledgement of expertise). This provided a basis for successive phases of co-construction and cooperation.

Opportunities for co-constructed research in this domain may also be arising in a period when civil service departments are under severe financial pressure and are looking to partnerships as a way to continue to deliver policy support with fewer in-house resources. This neatly coincides with the desire by SG and UK government for research to demonstrate how it contributes to positive societal impacts. When researchers can use research-based methods, tools and datasets to generate new insights from existing administrative datasets then this can be seen as reinforcing civil service capabilities rather than competing with them.

Threats inherent in co-construction are that researchers are participating in a political process where the institutions and norms are quite different. This meant adapting many aspects of how the research was conducted, making the outputs look less scientific despite their rigour. For example, the hypotheticodeductivist experimentalism common in natural sciences is neither a feasible nor an ethical basis for policymaking. Researchers also have to adapt to the research being directed rather than curiosity led so they have less control. They must also accept the limitations on the influence that science can, and in some cases is, allowed to have. Researchers also need to understand that timeliness is critical. Yet for researchers adapting to this milieu can create internal institutional threats if the outcomes of co-constructed policy support are not as highly valued as peer reviewed papers. The particular threats that can result from feedback from policy teams is explored in the next section.

\subsection{Value of co-construction to policy and other stakeholders}

The CAP analysis was one of six case studies chosen for in-depth analysis as part of the mid-programme review of the SRP, in addition to a wider questionnaire based study of science-policy interactions. An independent evaluation team who interviewed the researchers, analysts, policy makers, and other stakeholders conducted the case study. While the specific question of co-construction was not raised, it is possible to reinterpret stakeholder's responses against this background. The evaluators defined a ladder of impacts in terms of the perceived difficulty of achieving particular outcomes. These started from enhancing research capacity, through enduring connectivity, changes to attitudes/culture and concepts to instrumental change (in policy or practice). Overall the co-construction based approach was acknowledged by all parties as having resulted in instrumental change, i.e. it had informed decisions on regions and budgeting and reinforced negotiating positions in interactions between SG, the UK and Commission. This accorded well with public statements made by senior officials throughout the process. For the RESAS analysts the benefits in terms of additional and alternative research capacity were cited. For culture, the main interest of the evaluators was in assessing willingness to engage. This was well established between the research team and the main research partners but there was little evidence of increased willingness to engage on the part of direct stakeholders within some policy teams. This apparent gap was reinforced by a statement that there had been no conceptual change (i.e. new understanding or awareness), in particular that "the research had not led to new knowledge - as the policy team were already experts in the field". What was interesting was the same officials acknowledged that "the research activity played a role in the political process that gave credence to Scotland's negotiating position with [the lead department in UK government], where good quality evidence played an important part, and brought stakeholders on board". The evaluation team saw these instrumental outcomes as more difficult to achieve, so for researchers the evaluation is positive. Yet the evaluation team were surprised by the reluctance to acknowledge the research as having provided new knowledge. Several interpretations are possible. One is that by the time the evaluators asked the questions the researchers had been working with policy officials for over eight years (2006-14). What had perhaps been once new findings (explicit knowledge) may have been assimilated through the co-construction based research into expertise (or tacit knowledge). A second would be that policy officials may not have been aware of the new knowledge as having come from researchers as it was often incorporated into advice provided to them by RESAS analysts. A third interpretation is that as the influence of individuals within policymaking processes depends on 
perceptions of their expertise and soundness of judgement, perhaps the questioning inadvertently implied an unwelcome degree of displacement rather than support. It is perhaps worth considering if acknowledged conceptual change may be more challenging to achieve than instrumental change.

\section{DISCUSSION AND CONCLUSIONS}

This discussion includes only those ideas from the literature that particularly resonate with the authors' experiences in the CAP analysis. It is here, only possible to sketch a framework within which the practice of co-constructed can be evaluated. The intention is, however, to prompt further reporting of experiences by others in the field, to promote dialog and to serve as point of departure for further studies.

Co-construction is part of a wider discourse on how better to ensure that research generates outputs and outcomes that are relevant and useful in tackling major societal challenges (Matthews et al., 2011). Demonstration of the value of science conducted in support of public good outcomes is essential otherwise, given the trajectory of public finances, the support of such endeavours will be questioned. The CAP analysis has demonstrated the value of co-construction in building and maintaining productive relationships across the science-policy interface but perhaps in the process the researchers have ceased to be scientists?

Arguably the key insight from the CAP analysis team's previous years of research in the field of agricultural decision support systems development was that science-based tools must fit within socially mediated process of decision making and that they must complement rather than seek to displace judgement based systems (McCown, 2002). More recent research, in a related context, highlights social capital as the essential ingredient in the cooperation implied by co-construction (Flanigan and Sutherland, 2015). Here the key insights are that there must be sufficient initial similarities in values, attitudes and norms to generate bonds between participants and that over time, these ties are strengthened through mutually beneficial exchanges of knowledge or other resources. This capitals-based perspective identifies co-construction as one of the processes by which social capital is built and chimes strongly with the experiences of the CAP analysis team.

While the above speaks to the effective practice of co-construction, it does not necessarily explain its value in generating research outputs with greater influence. In that regard interdisciplinary research on environmental management by Leith et al. (2014), provides key insights from the field of science and technology studies. Here co-production (their term) is seen as the heart of creating usable knowledge, reshaping information and societal values and challenging scripts constructed to buttress privilege. Socially robust knowledge depends on salience, credibility and legitimacy (Cash et al., 2002) but we would argue that the most challenging of these for scientists is understanding the need for legitimacy. Scientists are used to assuming legitimacy based on epistemology, methodology and peer review. Yet none of these generate unchallengeable legitimacy beyond the walls of the academy. Indeed the very practices that make for high quality science (simplicity, objectivity and independence) leave research open to criticism as partial, utilitarian and utopian. The authors conclude that it is only by undertaking ongoing processes of co-construction and adaptive re-construction over the lifetime of research (such as practiced within the CAP analysis), that enables researchers to generate meaningful outputs in the circumstances of policymaking which is dominated by ambiguity, paradox, uncertainty and contested values (Nelson et al., 2008).

\section{ACKNOWLEDGEMENTS}

The research was funded by the Scottish Government strategic research programmes, 2006-11 and 2011-16. Particular thanks is due to Richard Murray and Neil Swanson of RESAS.

\section{REFERENCES}

Cash, D., Clark, W., Alcock, F., Dickson, N., Eckley, N., and Jager, J., Salience, Credibility, Legitimacy and Boundaries: Linking Research, Assessment and Decision Making., John F. Kennedy School of Government, Harvard University, 2002.

Flanigan, S. and Sutherland, L., Buying Access to Social Capital? From Collaboration to Service Provision in an Agricultural Co-operative, Sociologica Ruralis Early View - Online2015.

Matthews, K. B., Rivington, M., Blackstock, K. L., McCrum, G., Buchan, K., and Miller, D. G., Raising the bar? - The challenges of evaluating the outcomes of environmental modelling and software, Environmental Modelling and Software 26(3), 247-257, 2011.

McCown, R. L., Locating agricultural decision support systems in the troubled past and socio-technical complexity of 'models for management', Agricultural Systems 74(1), 11-25, 2002.

Nelson, R., Howden, M., and Smith, M. S., Using adaptive governance to rethink the way science supports Australian drought policy, Environmental Science \& Policy 11(7), 588-601, 2008. 